\title{
Dynamic medium of reference: A new theory of gravitation
}

\author{
Olivier Pignard ${ }^{\text {a) }}$ \\ 16 Boulevard du Docteur Cathelin, 91160 Longjumeau, France
}

(Received 25 May 2019; accepted 22 August 2019; published online 1 October 2019)

\begin{abstract}
The object of this article is to present a new theory based on the introduction of a nonmaterial medium which makes it possible to obtain a Preferred Frame of Reference (in the context of special relativity) or a Reference (in the context of general relativity), that is to say a dynamic medium of reference. The theory of the dynamic medium of reference is an extension of Lorentz-Poincaré's theory in the domain of gravitation in which instruments (clocks, rulers) are perturbed by gravitation and where only the measure of the speed of light always gives the same result. The presence of a massive body creates a centripetal flux of the medium, which has three fundamental effects: the dilatation of the period of material clocks, the contraction of the length of material rulers, and the slowdown of light. Thanks to the centripetal flux of the medium and these three effects, it is possible to find the correct expression of the deflection of a ray of light and the Shapiro delay. The dynamic medium of reference allows to establish a gravitational transformation and to find the fundamental equations of movement for light and matter. Hence, the theory of the dynamic medium of reference allows to find the main results of general relativity, but with important differences: The simultaneity is absolute, existence of the Preferred Frame of Reference, the physical reality is the universal present moment and not a global space-time (block-universe), light is slowed down by a gravitational field. (c) 2019 Physics Essays Publication.
\end{abstract}

[http://dx.doi.org/10.4006/0836-1398-32.4.422]

Résumé: L'objet de cet article est de présenter une nouvelle théorie basée sur l'introduction d'un milieu non-matériel qui permet d'obtenir un Référentiel Privilégié (dans le contexte de la relativité restreinte) ou une Référence (dans le contexte de la relativité générale), c'est-à-dire un milieu dynamique de référence. La théorie du milieu dynamique de référence est une extension de la théorie de Lorentz-Poincaré au domaine de la gravitation dans laquelle les instruments (horloges, règles) sont perturbées par la gravitation et seule la mesure de la vitesse de la lumière donne le même résultat. La présence d'un corps massif crée un flux centripète du milieu ce qui génère trois effets: La dilatation de la période des horloges matérielles; - La contraction de la longueur des règles matérielles; et - Le ralentissement de la lumière. Grâce au flux centripète du milieu et à ces trois effets, il est possible de trouver l'expression correcte de la déflection d'un rayon de lumière et de l'effet Shapiro. Le milieu dynamique de référence permet d'établir une transformation gravitationnelle et de trouver les équations fondamentales du mouvement pour la lumière et la matière. Ainsi, la théorie du milieu dynamique de référence permet de trouver les principaux résultats de la relativité générale, mais avec des différences importantes: La simultanéité est absolue, le Référentiel Privilégié existe, la réalité physique est le moment présent universel et non pas un espace-temps global (univers bloc), la lumière est ralentie par un champ gravitationnel.

Key words: General Relativity; Preferred Frame of Reference; Dynamic Medium of Reference; Lorentz-Poincaré's Theory; Simultaneity; Speed of Light.

\section{INTRODUCTION}

Up to now, general relativity is the best existing theory of gravitation and it provides very accurate values to many experiments and observations. ${ }^{1-3}$

Lorentz-Poincaré's theory competes with Einstein's special relativity. Although contested by some, Lorentz's theory is perfectly coherent. It has been defended by Henri Poincaré ${ }^{4}$ and Michel Lambert dedicates a whole chapter of

a)olivier_pacific@hotmail.fr
15 pages on Lorentz's theory in his book ${ }^{5}$ showing that this theory is perfectly acceptable.

The present article is the presentation of a theory that is an extension of Lorentz-Poincaré's theory in the domain of gravitation.

The proposed theory is based on the following concepts:

- Simultaneity is an absolute notion;

- Existence of a dynamic medium of reference;

- Existence of a reference time or privileged time, that is to say a universal present moment rather than a blockuniverse containing all past, present, and future events; 
- Centripetal flux of the medium created by a massive body; and

- The contraction of rulers and the dilatation of the periods of the clocks are physical effects due to the movement of rulers and clocks with regard to the medium or due to the movement of the medium created by a massive body.

Several renowned scientists have defended or defend most of these concepts.

Of course, Lorentz and Poincaré maintained their interpretation confronting Einstein's special relativity. John Bell also maintained the idea of returning to a prerelativistic theory, close to Lorentz's theory and the fact that the medium of propagation of light had been rejected based on erroneous arguments. ${ }^{6,7}$

Finally, in his book "Time Reborn," ${ }^{8}$ Lee Smolin claims the following arguments:

"The fact that it is always some moment in our perception, and that we experience that moment as one of a flow of moments, is not an illusion. It is the best clue we have to fundamental reality.

This means giving up the relativity of simultaneity and embracing its opposite: that there is a preferred global notion of time."

All these arguments bring support to the theory which will be developed in the present paper.

After the present introduction, Section II describes the fundamental characteristics of this medium. Section III provides three applications of the proposed theory corresponding to well-known tests of general relativity. Section IV gives the fundamental equations of movement in the theory of the dynamic medium of reference. Finally, the conclusion highlights the differences between this new theory and Einstein's relativity.

\section{THEORY OF THE DYNAMIC MEDIUM OF REFERENCE}

\section{A. Presentation of the dynamic medium of reference}

The proposed theory introduces a dynamic nonmaterial medium which is present in the whole Universe. The characteristics of this medium are:

- This medium enables one to deduce a Preferred Frame of Reference or rather a REFERENCE in the whole Universe and at all scales,

- This REFERENCE enables one to obtain a privileged time. The present moment is universal, that is to say the same in the whole Universe,

- This medium is also the medium of propagation of light,

- This medium verifies the principle of reciprocal action:

$\bigcirc$ The medium is distorted by matter and energy like the space-time of general relativity,

$\bigcirc$ The warping of this medium determines the trajectories of the particles (material particles and light particles).
The last two points explain that a ray of light is deflected by a massive body.

The presence of a massive body creates a flux of the medium (centripetal that is to say, directed toward the center of gravity of the massive body) of speed

$$
V_{\text {flux }}=\sqrt{\frac{2 G M}{r}}
$$

and acceleration

$$
\gamma_{\text {flux }}=\frac{G M}{r^{2}},
$$

where $r$ refers to the distance to the center of gravity of the massive body.

In the theory of the dynamic medium of reference, the factor

$$
K(r)=\left(1-\frac{2 G M}{c_{0}^{2} r}\right)^{-1 / 2}
$$

plays a role quite equivalent to the factor

$$
\gamma(V)=\left(1-\frac{V^{2}}{c_{0}^{2}}\right)^{-1 / 2} .
$$

- The material rulers are contracted by the factor $\gamma(V)$ due to their movement with regard to the dynamic medium of reference and they are contracted by the factor $K(r)$ due to a gravitational field (centripetal movement of the medium created by a massive body);

- The material clocks have their period dilated by a factor $\gamma(V)$ due to their movement with regard to the dynamic medium of reference and they have their period dilated by the factor $K(r)$ due to a gravitational field (centripetal movement of the medium created by a massive body).

However, there is a great difference between the two cases:

- In the case of the movement of a material ruler or clock, it is the movement of the ruler or the clock with regard to the Preferred Frame of Reference that creates the effect. The medium is not distorted.

- In the case of a gravitational field created by a massive body, the medium undergoes a centripetal flux increasing when one gets closer to the massive body. This flux, as a result, physically alters the length of the material rulers and the period of the material clocks. Another important point: as the medium is being physically altered by the presence of the massive body, this alteration plays the role of the curvature of space-time of general relativity.

In the case of a gravitational field, for a fix referential with regard to the massive body, the Preferred Frame of Reference moves at the same speed as a laboratory in free fall $V_{\mathrm{PFR}}=\sqrt{2 G M / r}$. 
The material clocks and the material rulers are then physically affected with the factor $\gamma\left(V_{\mathrm{PFR}}\right)=\left(1-\left(V_{\mathrm{PFR}}^{2} / c_{0}^{2}\right)\right)^{-1 / 2}$ which gives the factor of general relativity

$$
K(r)=\left(1-\frac{2 G M}{c_{0}^{2} r}\right)^{-1 / 2}
$$

This parallel between special relativity and gravitation is very important and justifies the claim that, for all dynamic phenomena in a gravitational field, the part due to the temporal effect is exactly the same as the part due to the spatial effect.

This is due to the fact that in special relativity, the factor used for the temporal part (clocks) is the same as the one used in the spatial part (rulers).

Remark: The centripetal flux of the medium is linked to the fundamental notion of the free fall referential of general relativity.

This concept of free fall referential is so essential that it is systematically used by Clifford Will in his book "Was Einstein right?", to explain the following phenomena in the presence of a gravitational field:

- Shifting of the clocks (Chapter 3),

- Shifting toward the red (redshift) of the light (Chapter 3),

- Deflection of the light (Chapter 4),

- Shapiro delay (Chapter 6).

Also, in their book "Relativité Générale," Julien Grain and Aurélien Barrau often use the free fall referential: ${ }^{9}$

"In a free fall referential, the correct laws must be the ones satisfying special relativity."

"From a geometric point of view, geodetics are the shorter lines between events of space-time. Physically, they are the trajectories of the test-masses in free fall."

\section{B. Material clock in a gravitational field}

As a result of what we have seen in Section II A, a material clock, of period $T_{0}$ when situated very far from all gravitational fields, undergoes a dilatation of its period of a factor $K(r)$ when situated at the distance $r$ from the center of gravity of a massive body, in such a way that its period is equal to

$$
T=T_{0} \cdot K(r) \text { with } K(r)=\left(1-\frac{2 G M}{c_{0}^{2} \cdot r}\right)^{-1 / 2}
$$

\section{Material ruler in a gravitational field}

Likewise, a material ruler, of length $L_{0}$ when situated very far from all gravitational fields, undergoes a contraction of a factor $K(r)$ when it is situated at the distance $r$ from the center of gravity of a massive body, in such a way that its length is equal to

$$
L=\frac{L_{0}}{K(r)} \text { with } K(r)=\left(1-\frac{2 G M}{c_{0}^{2} \cdot r}\right)^{-1 / 2}
$$

\section{Speed of light in the presence of a gravitational field}

\section{Speed of light for a radial trajectory}

From the two previous points, we can deduce the fundamental result:

The speed of light varies according to the distance

from the center of gravity of a massive body. light is

Indeed, very far from the massive body, the speed of

$$
c_{0}=\frac{L_{0}}{T_{0}} .
$$

On the other hand, at the distance $r$ from the center of gravity of the massive body, when light follows a radial trajectory, the speed of light actually has the following expression with regard to a referential linked to the massive body:

$$
c=\frac{L}{T}=\frac{L_{0} / K(r)}{T_{0} \cdot K(r)}=\frac{c_{0}}{K^{2}(r)}=c_{0}\left(1-\frac{2 G M}{c_{0}^{2} \cdot r}\right) .
$$

In the frame of radial trajectories, we can deduce an index of refraction of the medium due to gravitation

$$
n(r)=\frac{c_{0}}{c(r)}=\frac{1}{1-\frac{2 G M}{c_{0}^{2} \cdot r}}
$$

Light is therefore slower close to a massive body than very far from it.

This notable result allows one to find a good approximation of the Shapiro delay due to a massive body.

Another major point is that the measure of the speed of light results in the same value whatever distance we are from a massive body.

Indeed, if we own a ruler of length $L$ and a clock of period $T$, in such a way that the light covers the length of the ruler in a period of the clock, whatever the strength of the gravitational field we measure

$$
c=\frac{L}{T} \text {. }
$$

In fact, whether we are very far from all massive bodies (absence of gravitational field) or very close to the surface of a massive body, the light covers the length of the same ruler during the period of the same clock.

The measure provides an identical result of the speed of light, so apparently that light has a constant speed.

\section{Speed of light in a general case}

The expression of the speed of light found in Section II D 1 is only valid for a radial trajectory. 
For any kind of trajectory of light, the expression of speed is more complex and we are going to establish it.

The speed vector of light must be decomposed along two components which do not follow the same rule because the rulers are contracted by the factor $K(r)$ only when they are disposed along a radial of the massive body.

The radial component can be written

$$
c_{/ /}(r)=\frac{L_{/ /}}{T}=\frac{L_{0 / /} / K(r)}{T_{0} \cdot K(r)}=\frac{c_{0 / /}}{K^{2}(r)}=c_{0 / /} \cdot\left(1-\frac{k}{r}\right) .
$$

The ortho radial component can be written

$$
c_{\perp}(r)=\frac{L_{\perp}}{T}=\frac{L_{0 \perp}}{T_{0} \cdot K(r)}=\frac{c_{0 \perp}}{K(r)}=c_{0 \perp} \cdot \sqrt{1-\frac{k}{r}},
$$

where

$$
K(r)=\left(1-\frac{k}{r}\right)^{-1 / 2} \text { and } k=\frac{2 G M}{c_{0}^{2}} .
$$

Moreover, we have

$$
c_{0}=\sqrt{c_{0 / /}^{2}+c_{0 \perp}^{2}} .
$$

We name $\beta=(\overrightarrow{u / l}, \vec{c})$ the angle between the vector $\overrightarrow{u_{\|}}$and the speed vector of light $\vec{c}$.

The vector $\overrightarrow{c_{0}}$ represents the speed vector of light if there was not any massive body.

We can write

$$
\begin{gathered}
\left\{\begin{array}{l}
c_{/ /}(r)=c(r) \cos \beta \\
c_{\perp}(r)=c(r) \sin \beta
\end{array}\right\} \text { which gives us: } \\
\left\{\begin{array}{l}
c_{0 / /}=K^{2} c_{/ /}(r)=K^{2} \cdot c(r) \cos \beta \\
c_{0 \perp}=K \cdot c_{\perp}(r)=K \cdot c(r) \sin \beta
\end{array}\right\} .
\end{gathered}
$$

We also have

$$
c_{0}=\sqrt{c_{0 / /}^{2}+c_{0 \perp}^{2}}=K \cdot c(r) \sqrt{(K \cdot \cos \beta)^{2}+\sin ^{2} \beta} .
$$

Thus, we obtain the modulus of the speed vector of light

$$
c(r)=\frac{c_{0}}{K \sqrt{(K \cdot \cos \beta)^{2}+\sin ^{2} \beta}}
$$

which can also be written

$$
\begin{aligned}
& c(r)=\frac{c_{0}}{K \sqrt{1+\left(K^{2}-1\right) \cos ^{2} \beta}} \text { or even: } \\
& c(r)=c_{0} \sqrt{1-\frac{k}{r}} \cdot\left(1+\frac{k}{r-k} \cos ^{2} \beta\right)^{-1 / 2} .
\end{aligned}
$$

\section{E. The three fundamental effects of the theory of the dynamic medium of reference}

The three fundamental effects due to gravitation predicted by the theory of the dynamic medium of reference are the following:

- (1a) dilatation of the period of the clocks: $T=T_{0} \cdot K$,

- (1b) contraction of the length of the rulers: $L=L_{0} / K$,

- (2) distortion of the dynamic medium of reference: The gravitation creates centripetal flux of speed $V_{\text {flux }}=\sqrt{2 G M / r}$.

The two first effects have been noted (1a) and (1b) because:

- They are quite equivalent to the dilatation of durations and contraction of lengths of special relativity,

- For the light, the two cumulated effects imply the effect (1c): diminution of the speed of light in a gravitational field according to the rule

$$
c(r)=\frac{c_{0}}{K \sqrt{1+\left(K^{2}-1\right) \cos ^{2} \beta}} .
$$

The third effect (2) is specific to the domain of gravitation. Thus, it is an additional effect compared to special relativity (which corresponds to a nondistorted medium).

\section{F. New postulates of the theory of the dynamic medium of reference}

In the context of the theory of the dynamic medium of reference, the two Einstein's postulates become:

(1) The measure of the speed of light is constant in all referentials and in all directions, including in the presence of a gravitational field.

This implies that all instruments, in particular, the material clocks and the material rulers undergo physical effects due to their movement or the presence of a gravitational field in order to ensure a constant measure of the speed of light.

(2) All the inertial/Galilean referentials are equivalent for the expression of physical laws and there is a Preferred Frame of Reference or medium of reference in which the light really propagates at the speed $c 0$ and the material clocks and rulers do not undergo any physical effect of dilatation of their period or contraction of their length. This Preferred Frame of Reference or medium of reference makes it possible to define an absolute simultaneity and a privileged time everywhere in the Universe.

Important remark: In this theory, it is not question to come back to an absolute time as in Newton's theory where all the clocks of the Universe beat in unison whatever their speed and whatever the gravitational field.

In contrast, this theory proposes that all the material clocks (of same building) in all the parts of the Universe beat at exactly the same rhythm if and only if they are immobile 
with regard to the dynamic medium of reference. All these clocks provide the universal time of reference.

\section{G. Discussion on the principle of equivalence}

This part presents three objectives:

- to show that the principle of equivalence such as stated by Einstein in 1907 and 1911 can be used in the frame of Lorentz-Poincaré's theory in order to extend it to the domain of gravitation,

- to provide a new formulation of the principle of equivalence in the frame of the theory of the dynamic medium of reference and to show that it is sufficient to postulate the existence of the dynamic medium of reference to demonstrate the principle of equivalence, and

- to show that the formulation of the principle of equivalence in the frame of the theory of the dynamic medium of reference allows to establish that the inertia force due to a variation of the speed vector of a material object is fundamentally of the same nature that the gravitational force.

\section{Use of the principle of equivalence in the frame of Lorentz-Poincarés theory}

The equivalence principle ${ }^{10}$ allows us to state that a material clock undergoes exactly the same effect in the two following cases:

- The clock moves with an acceleration $\Gamma$ and with the speed $V$ with regard to the Preferred Frame of Reference. According to Lorentz-Poincaré's theory, the material clock undergoes a physical, real dilatation of its period,

- The clock is fixed with regard to a massive body of mass $M$ creating a gravitational field of acceleration $\Gamma$. According to the proposed interpretation, which is an extension of Lorentz-Poincaré's theory in the domain of gravitation, the material clock undergoes a physical, real dilatation of its period.

First, we are going to show that an object moving at the speed $V_{/ R}=-\sqrt{2 A / r}$ with regard to a referential $R$ ( $r$ indicates the distance from the origin $\mathrm{O}$ of the referential $R$ to the position of the object) undergoes an acceleration $\Gamma_{/ R}=-\left(A / r^{2}\right)$ (the signs are given with regard to a unit vector $\overrightarrow{u_{r}}$ going from the origin $\mathrm{O}$ toward the position of the object).

Starting from the speed $V=-\sqrt{2 A / r}$, the acceleration can be written

$$
\Gamma=\frac{d V}{d t}=\frac{d V}{d r} \frac{d r}{d t}=\frac{d V}{d r} \cdot V=\frac{1}{2} \frac{\sqrt{2 A}}{r^{3 / 2}}\left(-\sqrt{\frac{2 A}{r}}\right)=-\frac{A}{r^{2}} .
$$

So we can state the following important result:

For a clock moving with the speed $V_{/ \mathrm{PFR}}=-\sqrt{2 G M / r}$ with regard to the Preferred Frame of Reference PFR, this clock undergoes the acceleration $\Gamma_{/ \mathrm{PFR}}=-\left(G M / r^{2}\right)$.
According to Lorentz-Poincaré's theory, this material clock undergoes a physical dilatation of its period according to the formula

$$
T=\gamma \cdot T_{0} \text { with } \gamma=\left(1-\frac{V_{/ \mathrm{PFR}}^{2}}{c_{0}^{2}}\right)^{-1 / 2}=\left(1-\frac{2 G M}{c_{0}^{2} \cdot r}\right)^{-1 / 2} .
$$

$T_{0}$ indicates the period that the clock would have if it was immobile with regard to the Preferred Frame of Reference.

The equivalence principle ${ }^{10}$ allows us to claim that this clock undergoes the same effect being in the presence of a massive body of mass $M$ creating a gravitational field of acceleration $\Gamma=-\left(G M / r^{2}\right)$, that is to say, that its period undergoes a physical dilatation according to the formula

$$
T=K(r) \cdot T_{0} \text { with } K(r)=\left(1-\frac{2 G M}{c_{0}^{2} \cdot r}\right)^{-1 / 2} .
$$

$T_{0}$ indicates the period that the clock would have in the absence of gravitational field.

The equivalence principle ${ }^{10}$ allows us to state that a material ruler undergoes exactly the same effect in the two following cases:

- The ruler moves with an acceleration $\Gamma$ and with the speed $\mathrm{V}$ with regard to the Preferred Frame of Reference. According to Lorentz-Poincaré's theory, the material ruler undergoes a physical, real contraction of its length,

- The ruler is fixed with regard to a massive body of mass M creating a gravitational field of acceleration $\Gamma$. According to the proposed interpretation, which is an extension of Lorentz-Poincaré's theory in the domain of gravitation, the material ruler undergoes a physical, real contraction of its length.

We can state the following important result:

For a ruler moving with the speed $V_{/ \mathrm{PFR}}=-\sqrt{2 G M / r}$ with regard to the Preferred Frame of Reference PFR, this ruler undergoes the acceleration $\Gamma_{/ \mathrm{PFR}}=-\left(G M / r^{2}\right)$.

According to Lorentz-Poincaré's theory, this material ruler undergoes a physical contraction of its length according to the formula

$$
L=L_{0} / \gamma \text { with } \gamma=\left(1-\frac{V_{/ \mathrm{PFR}}^{2}}{c_{0}^{2}}\right)^{-1 / 2}=\left(1-\frac{2 G M}{c_{0}^{2} \cdot r}\right)^{-1 / 2} .
$$

$L_{0}$ indicates the length that the ruler would have if it was immobile with regard to the Preferred Frame of Reference.

The equivalence principle ${ }^{10}$ allows us to claim that this ruler undergoes the same effect being in the presence of a massive body of mass $M$ creating a gravitational field of acceleration $\Gamma=-\left(G M / r^{2}\right)$, that is to say, that its length undergoes a physical contraction according to the formula 


$$
L=L_{0} / K(r) \text { with } K(r)=\left(1-\frac{2 G M}{c_{0}^{2} \cdot r}\right)^{-1 / 2} \text {. }
$$

$L_{0}$ indicates the length that the ruler would have in the absence of gravitational field.

Conclusion: The principle of equivalence such as stated by Einstein in 1907 and 1911 is sufficient to establish the laws concerning the clocks and the rulers in a gravitational field and so to establish the speed of light in a gravitational field and finally to extend the Lorentz-Poincaré's theory to the domain of gravitation.

\section{New formulation of the principle of equivalence in the theory of the dynamic medium of reference}

In the frame of the theory of the dynamic medium of reference, the principle of equivalence is formulated in terms of speed and not acceleration and the two following statements are naturally equivalent:

- The effects on material clocks and rulers are due to their movement with regard to the medium of reference,

- The effects on material clocks and rulers in the presence of a massive body are due to the movement of the medium with regard to the massive body and so with regard to the clocks and the rulers.

This formulation is a justification of the principle of equivalence because it is obvious that the relative movement of the clocks and the rulers with regard to the medium is equivalent to the relative movement of the medium with regard to the clocks and the rulers.

In every case, it is the relative movement (speed) of the material clock and ruler with regard to the medium (which is the reference) which creates the effects (dilatation of the period of the material clock and contraction of the length of the material ruler).

\section{Inertia force}

In the theory of the dynamic medium of reference, the force of gravitation is due to the movement of the medium created by a massive body, which means that the force of gravitation is due to a variation of the speed vector of a material body with regard to the medium.

Thanks to the principle of equivalence established in the frame of the dynamic medium of reference, it is possible to state that, without gravitation field, all modification of the speed vector of a material object with regard to the medium of reference creates a force: It is the inertia force.

\section{APPLICATION OF THE THEORY OF THE DYNAMIC MEDIUM OF REFERENCE TO THREE TESTS OF GENERAL RELATIVITY}

This section details three applications of the theory of the dynamic medium of reference corresponding to three well-known tests of general relativity.

\section{A. Redshift of light}

The redshift or Einstein's effect is the frequential shifting of light or an electromagnetic signal due to a gravitational field.

This effect can be considered as a "corollary" of the slowdown of the clocks due to a gravitational field.

Indeed, if we consider two clocks, one located at a distance $r_{1}$ and the other at a distance $r_{2}$ from the center of gravity of a spherical body of mass $M$, the ratio between the periods of the two clocks is given by the following formula:

$$
\frac{T_{2}}{T_{1}}=\frac{K\left(r_{2}\right)}{K\left(r_{1}\right)} \text { with } K(r)=\left(1-\frac{2 G M}{c_{0}^{2} \cdot r}\right)^{-1 / 2} .
$$

Subsequently, it is sufficient to convert the periods at the points of emission and reception of light (or of the electromagnetic signal) in frequencies. A signal emitted at the frequency $F_{1}$ at the distance $r_{1}$ of the center of the spherical body is received at the distance $r_{2}$ at the frequency $F_{2}$ according to the formula:

$$
\frac{F_{1}}{F_{2}}=\frac{T_{2}}{T_{1}}=\frac{K\left(r_{2}\right)}{K\left(r_{1}\right)} \text { with } K(r)=\left(1-\frac{2 G M}{c_{0}^{2} \cdot r}\right)^{-1 / 2} .
$$

The proposed theory allows one to find the famous formula of the gravitational redshift.

Remark: If the distance $r_{2}$ is very large ("infinite"), it follows that $K\left(r_{2}\right)=1$ and $F_{2}=F_{0}$. Therefore, the previous formula can be written: $F_{1} / F_{0}=T_{0} / T_{1}=1 / K\left(r_{1}\right)$ or $F_{1}=F_{0} / K\left(r_{1}\right)$.

Therefore, at the surface of the Sun, the atoms will emit photons with a frequency $F_{1}$ smaller by a factor $K\left(r_{1}\right)$ with regard to $F_{0}$ which would be the frequency of the photons emitted by the same atoms without the presence of a gravitational field.

\section{B. Deflection of a ray of light \\ 1. Snell-Descartes law}

It has been known for many centuries that light is deflected when passing from one medium to another, which is called refraction.

If the first medium possesses a refraction index $n_{1}$, the second medium an index $n_{2}$, then the link between the incidence angle $i$ and the refracted angle $r$ is the famous SnellDescartes law which can be written: $n_{1} \cdot \sin (i)=n_{2} \cdot \sin (r)$.

If we use the two expressions $c_{1}=c_{0} / n_{1}$ and $c_{2}=c_{0} / n_{2}$, Snell-Descartes law can be written

$$
\frac{\sin (i)}{c_{1}}=\frac{\sin (r)}{c_{2}}
$$

If the speed $c_{2}$ is smaller than the speed $c_{1}$, then the angle of refraction is smaller than the incidence angle.

We can deduce that light is deflected in the direction where the speed of light is the smallest and we can thereby establish the following rule: 
A massive body has the effect of slowing down light when it gets closer to its center of gravity and of deflecting the ray of light oriented toward the massive body.

\section{Huygens-Fresnel's approach}

Using the Huygens-Fresnel's approach, the elementary angle of deflection of the ray of light is given by the following formula:

$d \alpha=(\partial c / \partial s) d t=(\partial c / \partial s)\left(d s / c_{0}\right)$, where $s$ represents the curvilinear abscissa covered by the ray of light, $d s$ an elementary part of this curvilinear abscissa, and $d t=d s / c_{0}$.

At a given point $M$ on the path of the ray of light, we call $\varphi$ the angle between the vector $\overrightarrow{d s}$ and the vector $\overrightarrow{d r}$.

We therefore have: $d r=\cos \varphi \cdot d s$ and thus $d s=d r / \cos \varphi$ and $\partial c / \partial s=(\partial c / \partial r) \cos \varphi$.

By taking into account this link between $d s$ and $d r$, we can write the elementary angle of deflection of the ray of light in the following way:

$$
d \alpha=\frac{1}{c_{0}} \frac{\partial c}{\partial s} d s=\frac{1}{c_{0}} \frac{\partial c}{\partial r} d r
$$

for a ray of light moving away from the massive body, that is to say, for which $(\partial c / \partial r)>0$.

The angle of deflection of the ray of light due to the slower speed of light is therefore given by the formula

$$
\alpha=\alpha_{1}+\alpha_{2} \text { with } \alpha_{1}=\int_{-\infty}^{r_{\text {min }}}-\frac{1}{c_{0}} \frac{\partial c}{\partial r} d r \text { and } \alpha_{2}=\int_{r_{\min }}^{+\infty} \frac{1}{c_{0}} \frac{\partial c}{\partial r} d r .
$$

Here, $r_{\min }$ represents the distance between the trajectory of the light and the center of gravity of the massive body when the light is closest to the massive body.

We have: $\alpha_{2}=\int_{r_{\min }}^{+\infty} \frac{1}{c_{0}} \frac{\partial c}{\partial r} d r=\frac{1}{c_{0}}[c(r)]_{r_{\min }}^{+\infty}=\frac{c(\infty)-c\left(r_{\min }\right)}{c_{0}}$ $=\frac{c_{0}-c\left(r_{\min }\right)}{c_{0}}$ because $c(\infty)=c_{0}$.

For obvious reasons of symmetry, we have $\alpha_{1}=\alpha_{2}$ (which is confirmed by the calculus of $\alpha_{1}$ ) so finally we obtain

$$
\alpha=2 \frac{c_{0}-c\left(r_{\min }\right)}{c_{0}} .
$$

\section{Remark:}

A more accurate expression of the elementary angle of deflection of the ray of light is the following:

$$
d \alpha=\frac{1}{c} \frac{\partial c}{\partial s} d s=\frac{1}{c} \frac{\partial c}{\partial r} d r
$$

We therefore obtain the angle of deflection of the ray of light due to the slowing down of speed of light

$$
\begin{aligned}
& \alpha=2 \int_{r_{\text {min }}}^{r_{\infty}} \frac{1}{c} \frac{\partial c}{\partial r} d r=2[\ln (c(r))]_{r_{\text {min }}}^{+\infty} \text { that is to say } \\
& \alpha=2 \ln \left(\frac{c_{0}}{c\left(r_{\text {min }}\right)}\right) .
\end{aligned}
$$

\section{Total angle of deflection of a ray of light}

a. The part due to the slowdown of the light

From Eq. (18), we can obtain the approached expression of the speed of light

$$
c(r) \approx c_{0}\left[1-\frac{k}{2 r}\left(1+\cos ^{2} \beta\right)\right] .
$$

At an infinite distance of the massive body we have: $c\left(r_{\infty}\right)=c_{0}$.

At the minimal distance of the massive body $r=r_{\min }$ we have $\beta \approx \pi / 2$ which shows that

$$
c\left(r_{\min }\right) \approx c_{0}\left(1-\frac{k}{2 r_{\min }}\right) .
$$

By using the relation $\alpha=2 \frac{c_{0}-c\left(r_{\text {min }}\right)}{c_{0}}$ we immediately obtain the angle of deflection

$$
\alpha=\frac{k}{r_{\min }}=\frac{2 G M}{c_{0}^{2} \cdot r_{\min }} .
$$

By using the relation $\alpha=2 \ln \left(c_{0} / c\left(r_{\min }\right)\right)$ we obtain

$$
\alpha=-2 \ln \left(1-\frac{k}{2 r_{\min }}\right) \approx \frac{k}{r_{\min }}=\frac{2 G M}{c_{0}^{2} \cdot r_{\min }} .
$$

\section{$b$. The part due to the distortion of the medium}

The part due to the distortion of the medium is caused by the fact that the medium of propagation of light undergoes a centripetal flux in the presence of a massive body.

This centripetal flux is even faster as we get closer to the massive body and its acceleration is expressed at the first approximation by the following formula:

$$
\gamma=\frac{G M}{r^{2}}
$$

The medium of propagation of light undergoing a centripetal flux, obviously this impacts a ray of light moving in its medium of propagation and this bends its trajectory in the direction of the center of gravity of the massive body.

The fundamental law is that a ray of light maintains its direction in a referential linked to its medium, that is to say, the Preferred Frame of Reference (we should say, the Reference since this referential is distorted seen by a referential linked to the massive body).

This implies that in a referential linked to the massive body, for an elementary moving of the ray of light $d L=c_{0} . d t$, the Preferred Frame of Reference moved in a centripetal way of the length $d L_{\mathrm{PFR}}=V_{\mathrm{PFR}} \cdot d t$ with $V_{\mathrm{PFR}}=\gamma_{\mathrm{PFR}} \cdot d t$ and $\gamma_{\mathrm{PFR}}=G M / r^{2}$.

For an almost rectilinear ray of light that we choose parallel to the axis of the abscissa in a landmark where the 
origin is placed at the center of gravity of the massive body, it undergoes an elementary deflection of an angle $d \alpha$ given by the following formula:

$\tan (d \alpha)=\left(d L_{\mathrm{PFR}} \cdot \cos (\pi / 2-\varphi) / d L\right)$ by taking $(r, \varphi)$ the polar coordinates of the current point $M$ and $(x, y)$ its Cartesian coordinates shown by the scheme below (Fig. 1).

The term $\cos ((\pi / 2)-\varphi)$ comes from the fact that the ray of light follows a trajectory almost parallel to the axis of the abscissa whereas the centripetal flux of the medium is on the radial of the massive body at the current point $M$.

The green rectangle symbolizes a free fall referential (for example, a laboratory or an elevator in free fall with a centripetal movement toward the center of gravity of the massive body). It also symbolizes a volume of the medium that undergoes a centripetal flux.

Let us pursue the calculus of the elementary angle of deflection

$$
\begin{aligned}
\tan (d \alpha)= & \frac{d L_{P F R} \cdot \cos \left(\frac{\pi}{2}-\varphi\right)}{d L} \\
= & \frac{V_{P F R} \cdot d t \cdot \cos \left(\frac{\pi}{2}-\varphi\right)}{c_{0} \cdot d t}=\frac{V_{P F R} \cdot \cos \left(\frac{\pi}{2}-\varphi\right)}{c_{0}} \\
= & \frac{\gamma_{P F R} \cdot d t \cdot \cos \left(\frac{\pi}{2}-\varphi\right)}{c_{0}} .
\end{aligned}
$$

$\tan (d \alpha)=\left(G M / r^{2}\right)\left(\sin (\varphi) / c_{0}\right) d t$ with $d L=c_{0} \cdot d t \approx d x$ and $\sin (\varphi)=y / r \approx R / r$ where $R$ refers to the distance from the closest point of the trajectory of the ray of light to the center of gravity of the massive body.

Whence finally the expression of the elementary angle

$$
d \alpha \approx \operatorname{atan}\left(\frac{G M R}{c_{0}^{2}} \frac{d x}{r^{3}}\right) \approx \frac{G M R}{c_{0}^{2}} \frac{d x}{r^{3}}
$$

By assuming that the ray of light comes from the infinite and goes to the infinite along the axis of abscissa, the total angle of deflection is given by the following formula:

$$
\begin{aligned}
\alpha & =\int d \alpha=\int_{-\infty}^{+\infty} \frac{G M R}{c_{0}^{2}} \frac{d x}{r^{3}}=\int_{-\infty}^{+\infty} \frac{G M R}{c_{0}^{2}} \frac{d x}{\left(x^{2}+y^{2}\right)^{3 / 2}} \\
& \approx \int_{-\infty}^{+\infty} \frac{G M R}{c_{0}^{2}} \frac{d x}{\left(x^{2}+R^{2}\right)^{3 / 2}} .
\end{aligned}
$$

By putting $u=x / R$ we have

$$
\begin{aligned}
\alpha & =\int_{-\infty}^{+\infty} \frac{G M R}{c_{0}^{2}} \frac{R \cdot d u}{\left((R \cdot u)^{2}+R^{2}\right)^{3 / 2}}=\frac{G M}{c_{0}^{2} R} \int_{-\infty}^{+\infty} \frac{d u}{\left(u^{2}+1\right)^{3 / 2}} \\
& =\frac{2 G M}{c_{0}^{2} R} \int_{0}^{+\infty} \frac{d u}{\left(u^{2}+1\right)^{3 / 2}} .
\end{aligned}
$$

We therefore use the well-known result $\int_{0}^{+\infty} d u /\left(u^{2}+1\right)^{3 / 2}=\left[u / \sqrt{u^{2}+1}\right]_{0}^{+\infty}=1$ which allows us to finally obtain the angle of deflection of the ray of light due to the distortion of the medium generating a centripetal flux

$$
\alpha_{\mathrm{flux}}=\frac{2 G M}{c_{0}^{2} R} .
$$

c. Total angle of deflection due to the slowing down of the speed of light and the flux of the medium

The total angle of deflection is the sum of the angle due to the slowing down of the speed of light and the angle due to the centripetal flux of the medium

$$
\alpha_{\text {total }}=\alpha_{\text {speed }}+\alpha_{\text {flux }}=\frac{2 G M}{c_{0}^{2} r_{\min }}+\frac{2 G M}{c_{0}^{2} R}=\frac{4 G M}{c_{0}^{2} r_{\min }} .
$$

We find once again the famous formula of general relativity.

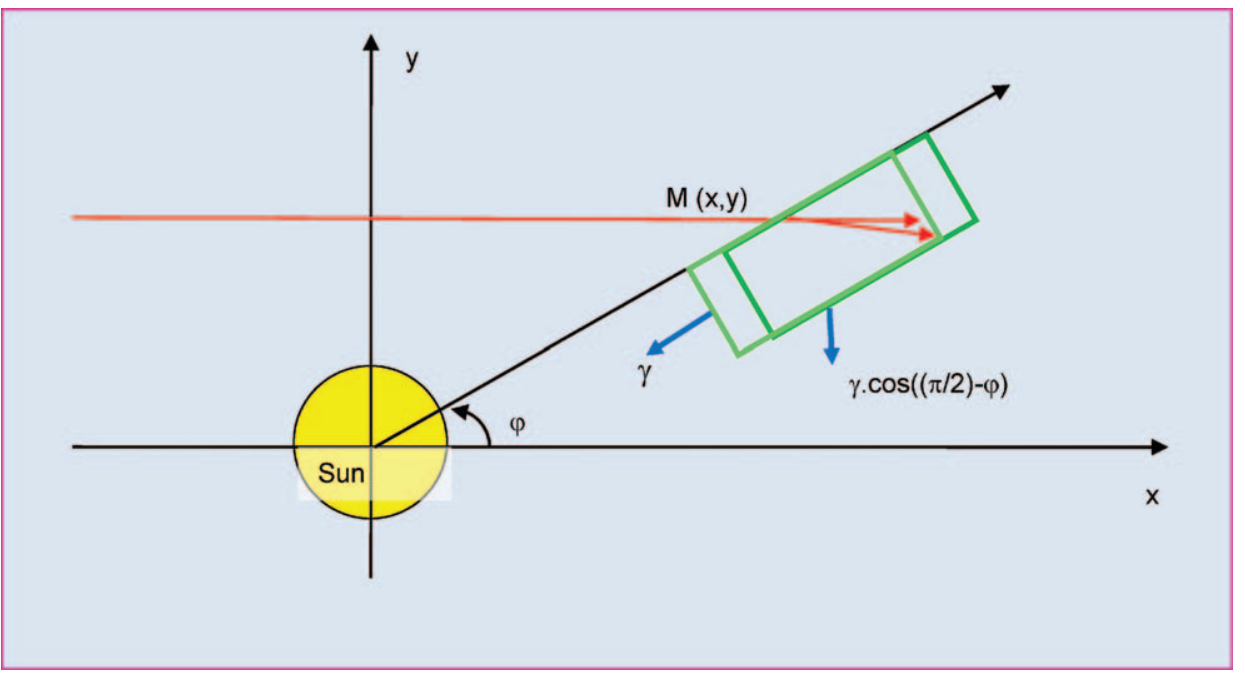

FIG. 1. Deflection of a ray of light by the Sun. 
Remark:

Still in the context of the radial case, by using the formula $n_{1} \cdot \sin (i)=n_{2} \cdot \sin (r)$ or the formula $\sin (i) / c_{1}=\sin (r) / c_{2}$ on successive elementary portions of the ray of light in a simulation, we obtain the exact value of the angle of deflection of the ray of light $\alpha=1.75 \mathrm{~s}$ of arc in the case of a ray of light approaching the Sun's surface.

\section{Shapiro effect}

\section{Radial Shapiro effect}

We study the Shapiro Effect on a radial of a massive body of mass $M$ and center of gravity $O$.

Since the trajectory of the light is radial, we will use the result established in Section II D 1 showing that light propagates more slowly near a massive body with a speed represented as

$$
c=c_{0}\left(1-\frac{k}{r}\right) \text { with } k=\frac{2 G M}{c_{0}^{2}} .
$$

We consider the time taken by light to travel from point $M_{1}\left(r_{1}=O M_{1}\right)$ to another point $M_{2}\left(r_{2}=O M_{2}\right)$, the three points $O, M_{1}, M_{2}$ being aligned in this order

$$
\begin{aligned}
\Delta t & =\int_{r_{1}}^{r_{2}} \frac{d r}{c(r)}=\frac{1}{c_{0}} \int_{r_{1}}^{r_{2}} \frac{d r}{1-\frac{k}{r}}=\frac{1}{c_{0}} \int_{r_{1}}^{r_{2}} \frac{r}{r-k} d r \\
& =\frac{1}{c_{0}} \int_{r_{1}}^{r_{2}} \frac{r-k+k}{r-k} d r=\frac{1}{c_{0}} \int_{r_{1}}^{r_{2}} d r+\frac{k}{c_{0}} \int_{r_{1}}^{r_{2}} \frac{d r}{r-k},
\end{aligned}
$$

which gives us

$$
\begin{aligned}
& \Delta t=\frac{r_{2}-r_{1}}{c_{0}}+\frac{k}{c_{0}}[\ln (r-k)]_{r_{1}}^{r_{2}} \text { that is to say } \\
& \Delta t=\frac{r_{2}-r_{1}}{c_{0}}+\frac{k}{c_{0}} \ln \left(\frac{r_{2}-k}{r_{1}-k}\right) .
\end{aligned}
$$

We immediately recognize the first term which is the duration in the Newtonian case $\Delta t_{\text {Newton }}=\left(r_{2}-r_{1}\right) / c_{0}$.
By ignoring $k$ with regard to $r_{1}$ and $r_{2}$, we finally obtain

$$
\Delta t=\Delta t_{\text {Newton }}+\frac{2 G M}{c_{0}^{3}} \ln \left(\frac{r_{2}}{r_{1}}\right) .
$$

Example: Case of a photon going from the surface of the Sun of radius $R_{S}$ and arriving at the surface of the Earth of radius $R_{T}$, by taking into account only the gravitational field due to the Sun $\left(D_{S T}\right.$ represents the distance Sun-Earth taken with regard to the centers of gravity)

$$
\Delta t=\Delta t_{\text {Newton }}+\frac{2 G M}{c_{0}^{3}} \ln \left(\frac{D_{S T}-R_{T}}{R_{S}}\right) .
$$

\section{Non radial Shapiro effect}

In this case, we consider the path of a ray of light going from point $M_{1}$ of Cartesian coordinates $\left(x_{1}, y_{1}\right)$ to point $M_{2}$ of Cartesian coordinates $\left(x_{2}, y_{2}\right)$ (Fig. 2).

The time taken by light to go from point $M_{1}$ to point $M_{2}$ is

$$
\Delta t=\int_{M_{1}}^{M_{2}} \frac{d x}{c(r)} .
$$

The speed of light is approximately expressed as

$$
\begin{aligned}
c(r) & \approx c_{0}\left[1-\frac{k}{2 r}\left(1+\cos ^{2} \beta\right)\right] \\
& =c_{0}\left[1-\frac{k}{2 r}\left(1+\cos ^{2}(\varphi+\alpha)\right)\right] \\
& \approx c_{0}\left[1-\frac{k}{2 r}\left(1+\cos ^{2} \varphi\right)\right]
\end{aligned}
$$

By using the formula $\cos \varphi=x / r$ we have $c(r) \approx c_{0}\left[1-(k / 2 r)-\left(k x^{2} / 2 r^{3}\right)\right]$ and therefore

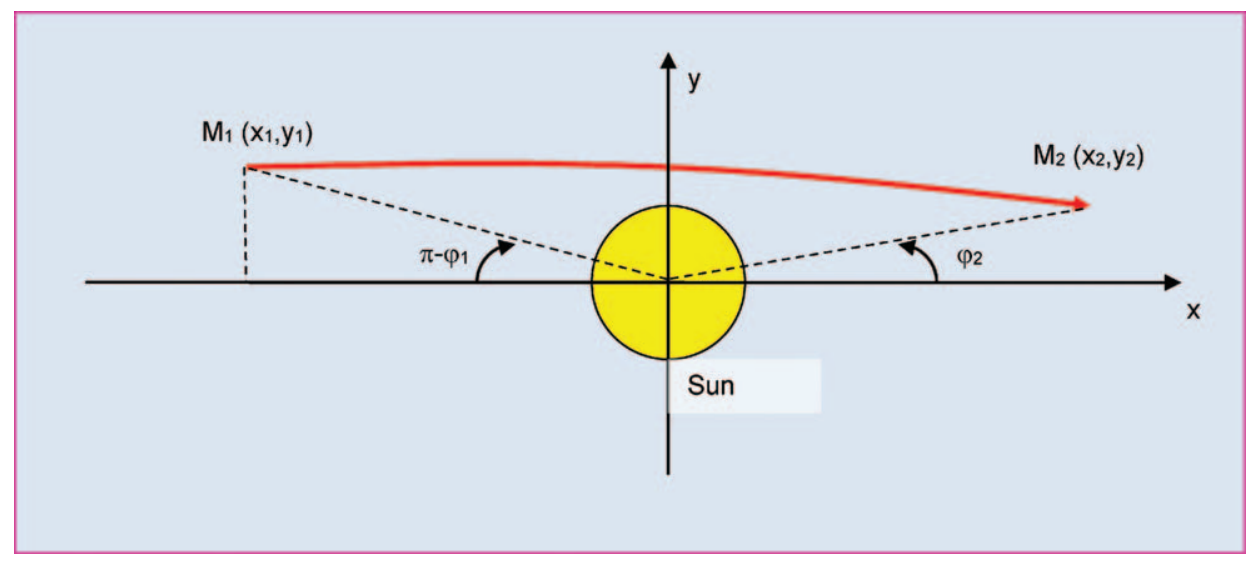

FIG. 2. Slowdown of light due to the Sun. 


$$
\begin{aligned}
\Delta t & =\int_{M_{1}}^{M_{2}} \frac{d x}{c(r)} \approx \frac{1}{c_{0}} \int_{x_{1}}^{x_{2}}\left[1+\frac{k}{2 r}\left(1+\cos ^{2} \varphi\right)\right] d x \\
& =\frac{1}{c_{0}} \int_{x_{1}}^{x_{2}} d x+\frac{k}{2 c_{0}} \int_{x_{1}}^{x_{2}} \frac{d x}{r}+\frac{k}{2 c_{0}} \int_{x_{1}}^{x_{2}} \frac{x^{2}}{r^{3}} d x .
\end{aligned}
$$

$\Delta t_{\text {Newton }}=1 / c_{0} \int_{x_{1}}^{x_{2}} d x=\left(x_{2}-x_{1}\right) / c_{0} \quad$ represents the duration of the travel of the ray of light in the Newtonian case.

If we define $f(x)=\ln (x+r)$ then we have

$$
\begin{aligned}
f^{\prime}(x) & =\frac{d f(x)}{d x}=\frac{1+\frac{d r}{d x}}{x+r}=\frac{1+\frac{d \sqrt{x^{2}+y^{2}}}{d x}}{x+r}=\frac{1+\frac{1}{2} \frac{2 x}{\sqrt{x^{2}+y^{2}}}}{x+r} \\
& =\frac{1+\frac{x}{r}}{x+r}=\frac{1}{r} .
\end{aligned}
$$

By writing $g(x)=x / r$ we have:

$g^{\prime}(x)=(1 / r)-x\left(\frac{d r / d x}{r^{2}}\right)=(1 / r)-\left(x^{2} / r^{3}\right)$.
Finally, we have

$$
\begin{aligned}
\Delta t & \approx \Delta t_{\text {Newton }}+\frac{k}{2 c_{0}} \int_{x_{1}}^{x_{2}}\left(2 \cdot f^{\prime}(x)-g^{\prime}(x)\right) d x \\
& \approx \Delta t_{\text {Newton }}+\frac{k}{2 c_{0}}\left[2 \cdot f\left(x_{2}\right)-2 \cdot f\left(x_{1}\right)-g\left(x_{2}\right)+g\left(x_{1}\right)\right] . \\
\Delta t & \approx \Delta t_{\text {Newton }}+\frac{k}{2 c_{0}}\left[2 \cdot \ln \left(\frac{x_{2}+r_{2}}{x_{1}+r_{1}}\right)-\frac{x_{2}}{r_{2}}+\frac{x_{1}}{r_{1}}\right]
\end{aligned}
$$

This formula can also be written:

$\Delta t \approx \Delta t_{\text {Newton }}+\frac{k}{2 c_{0}}\left[2 \cdot \ln \left(\frac{\left(r_{2}+x_{2}\right) \cdot\left(r_{1}-x_{1}\right)}{y_{1}^{2}}\right)-\frac{x_{2}}{r_{2}}+\frac{x_{1}}{r_{1}}\right]$. obtain

By using once again the relation $\cos \varphi=x / r$ we finally

$$
\begin{aligned}
\Delta t \approx & \Delta t_{\text {Newton }} \\
& +\frac{k}{2 c_{0}}\left[2 \cdot \ln \left(\frac{r_{1} \cdot r_{2}}{y_{1}^{2}}\left(1-\cos \varphi_{1}\right) \cdot\left(1+\cos \varphi_{2}\right)\right)\right. \\
& \left.+\cos \varphi_{1}-\cos \varphi_{2}\right] .
\end{aligned}
$$

TABLE I. Sum up of the results of the proposed theory and comparison with general relativity.

\begin{tabular}{ll}
\hline \hline General relativity & Theory of the dynamic medium of reference \\
\hline
\end{tabular}

A clock is affected by a gravitational field because of the curvature of space-time itself

$T=T_{\infty} \cdot K(r)$ with $K(r)=\left(1-\frac{2 G M}{c_{0}^{2} \cdot r}\right)^{-1 / 2}$

A ruler is affected by a gravitational field because of the curvature of space-time itself

$L=\frac{L_{\infty}}{K(r)}$ with $K(r)=\left(1-\frac{2 G M}{c_{0}^{2} \cdot r}\right)^{-1 / 2}$

Invariance of the speed of light

Frequency shift for a photon emitted at the surface of a massive body due to the curvature of space-time

$F=\frac{F_{\infty}}{K(r)}$

Deflection of light due to the curvature of space-time

$\alpha=\frac{4 G M}{c_{0}^{2} . r}$

Shapiro delay due to a longer path because of the curvature of space-time

Radial case: $\Delta t=\Delta t_{\text {Newton }}+\frac{2 G M}{c_{0}^{3}} \ln \left(\frac{r_{2}}{r_{1}}\right)$

General case: $\Delta t \approx \Delta t_{\text {Newton }}+\frac{2 G M}{c_{0}^{3}}\left[\ln \left(4 \frac{r_{1} \cdot r_{2}}{r_{\min }^{2}}\right)-1\right]$
A material clock is affected by the centripetal flux of the dynamic medium of reference

$T=T_{\infty} \cdot K(r)$ with $K(r)=\left(1-\frac{2 G M}{c_{0}^{2} \cdot r}\right)^{-1 / 2}$

A material ruler is affected by the centripetal flux of the dynamic medium of reference

$L=\frac{L_{\infty}}{K(r)}$ with $K(r)=\left(1-\frac{2 G M}{c_{0}^{2} \cdot r}\right)^{-1 / 2}$

The measure of the speed of light is constant, but light is really slowed down by a gravitational field

Radial case: $c=\frac{c_{0}}{K^{2}(r)}$

General case: $c=\frac{c_{0}}{K \sqrt{1+\left(K^{2}-1\right) \cos ^{2} \beta}}$

Frequency shift for a photon emitted at the surface of a massive body due to the dynamic medium of reference

$F=\frac{F_{\infty}}{K(r)}$

Deflection of light due to the dynamic medium of reference $\alpha=\frac{4 G M}{c_{0}^{2} \cdot r}$

Shapiro delay due to the slowdown of light by a gravitational field

Radial case: $\Delta t=\Delta t_{\text {Newton }}+\frac{2 G M}{c_{0}^{3}} \ln \left(\frac{r_{2}}{r_{1}}\right)$

General case: $\Delta t \approx \Delta t_{\text {Newton }}+\frac{2 G M}{c_{0}^{3}}\left[\ln \left(4 \frac{r_{1} \cdot r_{2}}{r_{\text {min }}^{2}}\right)-1\right]$ 
Case where $\varphi_{1} \approx \pi$ and $\varphi_{2} \approx 0$ (that is to say, $\cos \varphi_{1} \approx-1$ and $\cos \varphi_{2} \approx 1$ ).

By observing that $y_{1} \approx r_{\min }$ we have

$$
\Delta t \approx \Delta t_{\text {Newton }}+\frac{k}{c_{0}}\left[\ln \left(4 \frac{r_{1} \cdot r_{2}}{r_{\min }^{2}}\right)-1\right] \text {. }
$$

\section{Sum up of the obtained results and comparison with general relativity}

Table I sums up the main results of the Sections II and III.

\section{FUNDAMENTAL EQUATIONS OF MOVEMENT IN THE THEORY OF THE DYNAMIC MEDIUM OF REFERENCE}

The purpose of this section is to establish equations of movement, for photons and material particles in the frame of the theory of the dynamic medium of reference.

In order to do this, we are going to apply the following method:

- Establish equations of movement in a prerelativistic context (i.e., Newtonian),

- Apply to these equations a transformation due to the gravitation taking into account the results of Section II concerning the measure of durations and lengths.

\section{A. Gravitational transformation in the context of the theory of the dynamic medium of reference}

The purpose of this section is to establish a gravitational transformation whose principle is to start from prerelativistic (Newtonian) equations and to apply to them the results of Section II concerning the measure of durations and lengths.

We are going to establish the gravitational transformation for a speed expressed in polar coordinates $(r, \phi)$ since the flux of the medium is centripetal (i.e., directed toward the center of gravity of the massive body).

In polar coordinates, the speed can be written

$$
\begin{aligned}
\overrightarrow{V_{N}}= & V_{/ / N} \overrightarrow{u_{/ /}}+V_{\perp N} \overrightarrow{u_{\perp}} \\
& \text { with } V_{/ / N}=\left(\frac{d r}{d t}\right)_{N} \text { and } V_{\perp N}=\left(r \frac{d \phi}{d t}\right)_{N} .
\end{aligned}
$$

Thus, the modulus of the speed can be written

$$
V_{N}^{2}=V_{/ / N}^{2}+V_{\perp N}^{2}=\left(\frac{d r}{d t}\right)_{N}^{2}+\left(r \frac{d \phi}{d t}\right)_{N}^{2} .
$$

Taking into account the results of Section II concerning the measure of durations and lengths we have

- Radial speed: $V_{/ /}=V_{/ / N} \frac{L(r) / L_{\infty}}{T(r) / T_{\infty}}=V_{/ / N} \frac{\left(1-\frac{k}{r}\right)^{1 / 2}}{\left(1-\frac{k}{r}\right)^{-1 / 2}}$ that is to say

$$
V_{/ /}=K^{-2} V_{/ / N} \text { or } V_{/ / N}=K^{2} V_{/ /} \text {or }\left(\frac{d r}{d t}\right)_{N}=K^{2}\left(\frac{d r}{d t}\right) .
$$

- Ortho radial speed: $V_{\perp}=V_{\perp N} \frac{L(r) / L_{\infty}}{T(r) / T_{\infty}}=V_{\perp N} \frac{1}{\left(1-\frac{k}{r}\right)^{-1 / 2}}$
$=V_{\perp N} K^{-1}$ that is to say

$$
V_{\perp}=K^{-1} V_{\perp N} \text { or } V_{\perp N}=K V_{\perp} \text { or }\left(r \frac{d \phi}{d t}\right)_{N}=K\left(r \frac{d \phi}{d t}\right),
$$

where $K(r)=\left(1-\frac{2 G M}{c_{0}^{2} r}\right)^{-1 / 2}$ and $k=\frac{2 G M}{c_{0}^{2}}$.

Finally, we obtain the first fundamental equation

$$
\begin{aligned}
V_{N}^{2} & =V_{/ / N}^{2}+V_{\perp N}^{2} \\
& =\left(\frac{d r}{d t}\right)_{N}^{2}+\left(r \frac{d \phi}{d t}\right)_{N}^{2}=\left(K^{2} V_{/ /}\right)^{2}+\left(K V_{\perp}\right)^{2} \\
& =\left(K^{2} \frac{d r}{d t}\right)^{2}+\left(K r \frac{d \phi}{d t}\right)^{2} .
\end{aligned}
$$

As in the proposed theory, the gravitation is due to a flux of the medium directed toward the center of gravity of the massive body, it is considered that the conservation of the angular momentum is valid

$$
r_{N}^{2}\left(\frac{d \phi}{d t}\right)_{N}=r_{N}\left(r \frac{d \phi}{d t}\right)_{N}=r_{N} V_{\perp N}=A=\text { constant. }
$$

By using the expressions $V_{\perp N}=K \cdot V_{\perp}$ and $r_{N}=K \cdot r$, we obtain the second fundamental equation

$$
r_{N} V_{\perp N}=K^{2} \cdot r \cdot V_{\perp}=K^{2} r^{2} \frac{d \phi}{d t}=A .
$$

\section{B. Photon}

In the context of the theory of the dynamic medium of reference, the approach is the following:

- start from the equation $c_{\text {photon }}=c_{0}$,

- write this equation in polar coordinates in the prerelativist (Newtonian) context,

- transform this last equation using the gravitational transformation.

The equation $c_{\text {photon }}^{2}=c_{0}^{2}$ can be written in polar coordinates: $V_{N}^{2}=(d r / d t)_{N}^{2}+(r(d \phi / d t))_{N}^{2}=c_{0}^{2}$.

By using the gravitational transformation we obtain the fundamental formula

$$
\left(K^{2} \frac{d r}{d t}\right)^{2}+\left(K r \frac{d \phi}{d t}\right)^{2}=c_{0}^{2}
$$


In the frame of general relativity, this equation can be obtained thanks to the Lagrangian.

For a null geodesic (case of the photon), the first integral of the geodesic equations is

$$
L=K^{2}\left[c_{0}^{2}-\left(K^{2} \frac{d r}{d t}\right)^{2}-\left(K r \frac{d \phi}{d t}\right)^{2}\right]=0
$$

where $L$ indicates the Lagrangian (see Ref. 11, Chap. 9).

It is possible to interpret the previous result in the following way:

- In the medium of propagation (medium of reference) the speed of a photon is $\overrightarrow{c_{\text {photon }}}=\overrightarrow{c_{0}}$.

- In a referential linked to the massive body (with for origin the center of gravity of the massive body) the speed of the photon is such: $\left(K^{2}(d r / d t)\right)^{2}+(K r(d \phi / d t))^{2}=c_{0}^{2}$.

We are going to carry on the calculations in order to obtain an equation in $d r / d \phi$.

By using the second fundamental equation (52) in Eq. (53) we obtain

$$
\left(K^{2} \frac{d r}{d t}\right)^{2}+\left(\frac{A}{K r}\right)^{2}=c_{0}^{2}
$$

By using the formula $d r / d t=(d r / d \phi)(d \phi / d t)=$ $\left(A / K^{2} r^{2}\right)(d r / d \phi)$ we obtain

$$
\left(\frac{A}{r^{2}} \frac{d r}{d \phi}\right)^{2}+\left(\frac{A}{K r}\right)^{2}=c_{0}^{2} \text { or }\left(\frac{1}{r^{2}} \frac{d r}{d \phi}\right)^{2}+\left(\frac{1}{K r}\right)^{2}=\left(\frac{c_{0}}{A}\right)^{2}
$$

If we put $u=1 / r$ we obtain: $d r / d \phi=-\left(1 / u^{2}\right)(d u / d \phi)$ and so we get

$$
\begin{aligned}
\left(\frac{d u}{d \phi}\right)^{2}+K^{-2} u^{2}= & \left(\frac{c_{0}}{A}\right)^{2} \\
& \text { or }\left(\frac{d u}{d \phi}\right)^{2}+u^{2}-\frac{2 G M}{c^{2}} u^{3}=\left(\frac{c_{0}}{A}\right)^{2} .
\end{aligned}
$$

If we derive this expression with respect to $\phi$ and then divide it by $2(d u / d \phi) \neq 0$ we obtain

$$
\frac{d^{2} u}{d \phi^{2}}+u=\frac{3 G M}{c^{2}} u^{2}
$$

This equation makes it possible to determine the deflection of rays of light by the Sun and also by cluster of galaxies (gravitational lens, gravitational mirage, Einstein's ring).

Case of the radial trajectory:

In this case, we have the very simple expression

$$
\left(K^{2} \frac{d r}{d t}\right)^{2}=c_{0}^{2} \text { or also } K^{2} \frac{d r}{d t}= \pm c_{0} \text {. }
$$

Finally, we have

$\frac{d r}{d t}= \pm K^{-2} c_{0}= \pm\left(1-\frac{2 G M}{c_{0}^{2} r}\right) c_{0}$.

We could have directly found this formula by using $(d r / d t)_{N}^{2}=c_{0}^{2}$ and $(d r / d t)_{N}=K^{2}(d r / d t)$.

\section{Material particle}

In the context of the theory of the dynamic medium of reference, the approach is the following:

- Start from the equation

$$
\vec{\Gamma}_{\text {particule }}=\vec{\Gamma}_{\text {flux }} \text { with } \vec{\Gamma}_{f \text { lux }}=-\frac{G M}{r^{2}} \overrightarrow{u / /}_{\longrightarrow}
$$

- Write this equation in polar coordinates in a prerelativist (Newtonian) context, that is to say $\left(\vec{\Gamma}_{\text {particule }}\right)_{N}=\left(\vec{\Gamma}_{\text {flux }}\right)_{N}$ an equation in which the acceleration of the flux in a prerelativistic context has the approximate expression $\left(\vec{\Gamma}_{\text {flux }}\right)_{N} \approx-\left(G M / r_{N}^{2}\right) \overrightarrow{u / /}$,

- Integrate this equation in order to obtain an equation with speed,

- Transform this last equation using the gravitational transformation.

In polar coordinates the acceleration can be written:

$$
\overrightarrow{\Gamma_{N}}=\Gamma_{/ / N} \overrightarrow{u_{/ /}}+\Gamma_{\perp N} \overrightarrow{u_{\perp}}
$$

with $\Gamma_{/ / N}=\left(d^{2} r / d t^{2}\right)_{N}-r_{N}(d \phi / d t)_{N}^{2}$ and $\Gamma_{\perp N}=r_{N}\left(d^{2} \phi / d t^{2}\right)_{N}+2(d r / d t)_{N}(d \phi / d t)_{N}$.

The equation $\left(\vec{\Gamma}_{\text {particule }}\right)_{N}=\left(\vec{\Gamma}_{\text {flux }}\right)_{N} \approx-\left(G M / r_{N}^{2}\right) \overrightarrow{u_{/ /}}$ can be written

$$
\left(\frac{d^{2} r}{d t^{2}}\right)_{N}-r_{N}\left(\frac{d \phi}{d t}\right)_{N}^{2} \approx-\frac{G M}{r_{N}^{2}}
$$

The movement of the particle being with a radial acceleration, the conservation of the angular momentum (deduced from $\left.\Gamma_{\perp N}=0\right)$ is valid: $r_{N}^{2}(d \phi / d t)_{N}=A=$ constant.

Equation (61) can be written as

$$
\left(\frac{d^{2} r}{d t^{2}}\right)_{N}-\frac{A^{2}}{r_{N}^{3}} \approx-\frac{G M}{r_{N}^{2}}
$$
obtain

After multiplying this last equation by $2(d r / d t)_{N}$ we

$$
2\left(\frac{d r}{d t}\right)_{N}\left(\frac{d^{2} r}{d t^{2}}\right)_{N}-2 \frac{A^{2}}{r_{N}^{3}}\left(\frac{d r}{d t}\right)_{N} \approx-2 \frac{G M}{r_{N}^{2}}\left(\frac{d r}{d t}\right)_{N} .
$$

After integrating this last equation, we obtain

$(d r / d t)_{N}^{2}+\left(A^{2} / r_{N}^{2}\right) \approx\left(2 G M / r_{N}\right)+C^{2}$ where $C^{2}$ is an integration constant taking into account the initial conditions in position and speed of the particle. 
Using the relation $r_{N}^{2}(d \phi / d t)_{N}=A$ we can rewrite the previous equation in this way

$$
\left(\frac{d r}{d t}\right)_{N}^{2}+\left(r \frac{d \phi}{d t}\right)_{N}^{2} \approx \frac{2 G M}{r_{N}}+C^{2} \approx\left(V_{\text {flux }}^{2}\right)_{N}+C^{2} .
$$

By using the gravitational transformation, we obtain the following fundamental formula:

$$
\left(K^{2} \frac{d r}{d t}\right)^{2}+\left(K r \frac{d \phi}{d t}\right)^{2}=\frac{2 G M}{r}+C^{2}=V_{\text {flux }}^{2}+C^{2} .
$$

In the context of general relativity, this equation can be obtained thanks to the Lagrangian.

For a nonnull geodesic, the first integral of the geodesic equations is

$L=c_{0}^{2}$ which can be written

$$
c_{0}^{2}-L=K^{2}\left[\left(K^{2} \frac{d r}{d t}\right)^{2}+\left(K r \frac{d \phi}{d t}\right)^{2}-\frac{2 G M}{r}-C^{2}\right]=0,
$$

where $L$ indicates the Lagrangian (see Ref. 11, Chap. 9).

It is possible to interpret the previous result in the following way:

- In the dynamic medium of reference, the speed of a material particle is $\overrightarrow{V_{\text {particule }}}=\overrightarrow{V_{0}}$,

- In a referential linked to the massive body (with for origin the center of gravity of the massive body) the speed of a particle is such: $\left(K^{2}(d r / d t)\right)^{2}+(K r(d \phi / d t))^{2}=(2 G M / r)+C^{2}$.

Remark: In the case of a material particle with a null speed at an infinite range $(r=\infty)$, we have $d r / d t=0$, $r . d \phi / d t=A / r=0, K=1$ which implies $C=0$.

So, the equation can be written

$$
\left(K^{2} \frac{d r}{d t}\right)^{2}+\left(K r \frac{d \phi}{d t}\right)^{2}=\frac{2 G M}{r}=V_{\text {flux }}^{2} .
$$

We consider that the expression of the speed of the flux $\vec{V}_{\text {flux }}=-\sqrt{(2 G M / r)} \overrightarrow{u_{\| /}}$, which was only an approximation in a prerelativistic context, is accurate in the context of the theory of the dynamic medium of reference.

We are going to carry on the calculations in order to obtain an equation in $d r / d \phi$.

Using the second fundamental equation (52) in Eq. (64) we obtain

$$
\left(K^{2} \frac{d r}{d t}\right)^{2}+\left(\frac{A}{K r}\right)^{2}=\frac{2 G M}{r}+C^{2} .
$$

Using the formula $(d r / d t)=(d r / d \phi)(d \phi / d t)=$ $\left(A / K^{2} r^{2}\right)(d r / d \phi)$ we obtain

$$
\begin{aligned}
& \left(\frac{A}{r^{2}} \frac{d r}{d \phi}\right)^{2}+\left(\frac{A}{K r}\right)^{2}=\frac{2 G M}{r}+C^{2} \text { or furthermore } \\
& \left(\frac{1}{r^{2}} \frac{d r}{d \phi}\right)^{2}+\left(\frac{1}{K r}\right)^{2}=\frac{2 G M}{A^{2} r}+\frac{C^{2}}{A^{2}} .
\end{aligned}
$$

Putting $u=1 / r$ we obtain $d r / d \phi=-\left(1 / u^{2}\right)(d u / d \phi)$ which gives us

$$
\begin{aligned}
\left(\frac{d u}{d \phi}\right)^{2}+K^{-2} u^{2}= & \frac{2 G M}{A^{2}} u+\frac{C^{2}}{A^{2}} \text { or } \\
& \left(\frac{d u}{d \phi}\right)^{2}+u^{2}-\frac{2 G M}{c^{2}} u^{3}=\frac{2 G M}{A^{2}} u+\frac{C^{2}}{A^{2}} .
\end{aligned}
$$

If we derive this expression with respect to $\phi$ and then divide it by $2(d u / d \phi) \neq 0$ we obtain

$$
\frac{d^{2} u}{d \phi^{2}}+u=\frac{G M}{A^{2}}+\frac{3 G M}{c^{2}} u^{2} .
$$

This equation allows one to determine the trajectory of the planets of the solar system and in particular, the precession of Mercury's perihelion.

Case of a radial trajectory of a material particle with a null speed at an infinite range

In this case, we have the very simple expression

$$
\left(K^{2} \frac{d r}{d t}\right)^{2}=\frac{2 G M}{r} \text { or also } K^{2} \frac{d r}{d t}= \pm \sqrt{\frac{2 G M}{r}}= \pm V_{\text {flux }} \text {. }
$$

Finally, we have

$$
\frac{d r}{d t}= \pm K^{-2} \sqrt{\frac{2 G M}{r}}= \pm\left(1-\frac{2 G M}{c_{0}^{2} r}\right) \sqrt{\frac{2 G M}{r}} .
$$

We could have directly found this formula by using

$$
\left(\frac{d r}{d t}\right)_{N}^{2} \approx \frac{2 G M}{r} \text { and }\left(\frac{d r}{d t}\right)_{N}=K^{2}\left(\frac{d r}{d t}\right) .
$$

\section{CONCLUSION}

The present article proposes a new theory with the following features:

- A material ruler is physically contracted due to its motion with regard to the medium of reference;

- A material clock has its period physically dilated due to its motion with regard to the medium of reference;

- A material ruler is physically contracted due to the movement of the medium created by a massive body;

- A material clock has its period physically dilated due to the movement of the medium created by a massive body; 
- Light is slowed down by the gravitational field (centripetal flux of the medium) created by a massive body.

According to the previous features, the theory of the dynamic medium of reference allows to obtain the following results:

- deviation of light by the gravitational field (centripetal flux of the medium) created by a massive body,

- Shapiro delay due to the gravitational field (centripetal flux of the medium) created by a massive body,

- gravitational transformation which allows to obtain the equations of movement for photons and material particles.

The concepts, the starting hypothesis and postulates, the physical phenomenon explaining gravitation and above all the interpretation differ greatly between the theory of the dynamic medium of reference and general relativity.

In the context of general relativity, gravitation is a geometric modification of space-time. A massive body curves the space-time.

In the context of the theory of the dynamic medium of reference, gravitation is a modification of the medium. A massive body affects the medium by generating flux directed toward the center of gravity of the massive body.

Table II gives the main differences between the two theories.

The theory of the dynamic medium of reference is an extension of Lorentz-Poincaré's theory in the domain of gravitation where instruments (rulers and clocks) are distorted by gravitation and it is only the measure of the speed of light which always gives the same result, the light being really slowed down by a gravitational field.

Most well-known scientists working on general relativity think that physical reality is global space-time, called blockuniverse, which contains all past, present and future events. All events exist eternally.

In the context of the theory of the dynamic medium of reference, the only physical reality is the universal present moment, where travels in the past are impossible because the past no longer exists, the future does not exist yet and is open.

One can choose one or the other theory.

\section{ACKNOWLEDGMENTS}

I would like to thank Mr. Roger Balian, member of the French Science Academy, for his pertinent remarks and advice concerning my document. I would also like to thank Mr. Michel Barreteau, researcher at Thales Research \& Technology, for reading and correcting this article.

\section{APPENDIX A: A POSSIBLE DESCRIPTION OF THE DYNAMIC MEDIUM OF REFERENCE}

This part gives a possible description of the dynamic medium of reference.

The theory of the dynamic medium of reference is based on Le Sage theory, but it adds many deep changes and evolutions.

Numerous scientists have studied Le Sage theory. Just to mention a few of them:

Newton, Huygens, Leibniz, Euler, Laplace, Lord Kelvin, Maxwell, Lorentz, Hilbert, Darwin, Poincaré, and Feynman.

Henri Poincaré has studied this theory and written a synthesis in Science et Méthode. ${ }^{4}$

Poincaré sums up the principle of Le Sage theory like this:

"It is proper to establish a parallel between these considerations and a theory proposed a long time ago in order to explain the universal gravitation. Let's suppose that, in the interplanetary spaces, very tiny particles move in all directions, with very high speeds. A single body in the space will not be affected, apparently, by the impact of these corpuscles, since these impacts are equally divided in all directions. But, if two bodies A and B are in the space, the body B will play the role of a screen and will intercept a part of these corpuscles which would have hit A. Then, the impacts received by $\mathrm{A}$ in the opposite direction of the one of $\mathrm{B}$, will

TABLE II. Main differences between general relativity and the theory of the dynamic medium of reference.

\begin{tabular}{|c|c|}
\hline General relativity & Theory of the dynamic medium of reference \\
\hline \multirow[t]{2}{*}{ Simultaneity relative to the observer ${ }^{12,13}$} & Absolute simultaneity \\
\hline & Independent of the observer \\
\hline $\begin{array}{l}\text { Existence of a block-universe }{ }^{13} \\
\text { (containing all past, present, and future events) }\end{array}$ & $\begin{array}{l}\text { The only physical reality is the universal present moment, the past } \\
\text { does not exist any longer, the future does not exist yet and is open }\end{array}$ \\
\hline The aether does not exist & Existence of the medium of propagation of light \\
\hline No Preferred Frame of Reference & Existence of the Preferred Frame of Reference ${ }^{6,7}$ \\
\hline Invariance of the speed of light ${ }^{12}$ & $\begin{array}{l}\text { The measure of the speed of light is constant, but light is really slowed } \\
\text { down by a gravitational field }\end{array}$ \\
\hline Gravitation $=$ geometric modification of space-time & $\begin{array}{l}\text { Gravitation }=\text { modification of the medium (flux directed toward the } \\
\text { center of gravity of the massive body) }\end{array}$ \\
\hline $\begin{array}{l}\text { A gravitational wave is a small deformation of space time } \\
\text { propagating at the speed of light }\end{array}$ & $\begin{array}{l}\text { A gravitational wave is a small deformation of the medium propagat- } \\
\text { ing at the speed of light }\end{array}$ \\
\hline The speed of propagation of gravitation is the speed of light & $\begin{array}{l}\text { The speed of propagation of gravitation is much greater than the speed } \\
\text { of light }\end{array}$ \\
\hline
\end{tabular}


not have compensation any longer, or will be imperfectly compensated, and they will push A toward B. Such is Le Sage theory."

It is possible to demonstrate rather easily that the "push" is inversely proportional to the square distance between the two bodies (like the Newton law).

One can also demonstrate that, if the corpuscles are very tiny, the push is approximately proportional to the number of nucleons and so the mass of the body, and not the apparent surface of the body.

Moreover, only a tiny fraction of corpuscles hits the atoms of the body, which explains that the push (the gravitational force) is so weak.

The main evolutions of the theory of the dynamic medium of reference versus Le Sage theory are the following:

- one must not use the notion of impact with the corpuscles,

- one must consider that the corpuscles are nonmaterial and constitute a medium,

- the total energy of an entity is the sum of its kinetic energy of translation and of its kinetic energy of rotation about itself, and

- fundamental law: conservation of the total energy of an entity: the total energy of one entity remains constant: $E_{\text {total }}=E_{\text {translation }}+E_{\text {rotation }}=$ constant.

Afterward, I will call these entities gravitons (but these gravitons have nothing to do with the graviton of spin 2 of quantum mechanics).

If the total energy of the gravitons remains constant, then the gravitons do not give energy to the atoms of the Earth and so do not raise the temperature of the Earth.

It is postulated that the gravitons which interact with the atoms of the Earth lose some of their kinetic energy of translation which turns into kinetic energy of rotation. The gravitons which interact with the atoms of the Earth lose a part of their translation speed and win some rotation speed.

So the Earth would be a huge "transformer" of "standard gravitons" in "gravitons-spin."

This physical phenomenon has no effect on Earth (at least not the elevation of temperature), but it has an effect on the medium.

The medium undergoes a centripetal flux due to the presence of the Earth.

Indeed, let us consider a reference frame at the surface of the Earth and an elementary volume linked to it.

If one measures the speed vectors of all the gravitons in this elementary volume, the average of the speed vectors gives a resulting speed vector which is centripetal (because the gravitons-spin coming from the ground have a smallest translation speed than the standard gravitons coming from the sky).

One can demonstrate that the acceleration of the flux has the following expression $\overrightarrow{\gamma_{\text {flux }}}=-\left(G M / r^{2}\right) \overrightarrow{u_{r}}$ from which one can deduce that the centripetal speed of the flux at a distance $r$ from the center of gravity of the Earth of mass $M$ is equal to (measured in a reference frame $R$ linked to the Earth):

$$
\overrightarrow{C_{G / R}}=\frac{\sum_{i=1}^{N_{G}} \overrightarrow{V_{G / R}}}{N_{G}}=\overrightarrow{V_{\text {flux }}}=-\sqrt{\frac{2 G M}{r}} \overrightarrow{u_{r}} .
$$

The demonstration of the link between the acceleration and the speed of the flux is the following:

If we derivate the acceleration we obtain

$\gamma_{\text {flux }}=\frac{d V}{d t}=\frac{d V}{d r} \frac{d r}{d t}=\frac{d V}{d r} \cdot V=\frac{1}{2} \frac{d}{d r}\left(V^{2}\right)$ so we have: $V_{\text {flux }}^{2}=\int_{+\infty}^{r} 2 \gamma_{\text {flux }} d r$.

Using the expression $\gamma_{\text {flux }}=-\left(G M / r^{2}\right)$ we obtain: $V_{\text {flux }}^{2}=-\int_{+\infty}^{r}\left(2 G M / r^{2}\right) d r=[2 G M / r]_{+\infty}^{r}=2 G M / r$.

Finally, we obtain the expression of the speed of the flux: $\overrightarrow{V_{\text {flux }}}=-\sqrt{(2 G M / r)} \overrightarrow{u_{r}}$.

Remark: The speed of the flux is zero at an infinite distance of the massive body creating the gravitational field $\left(\mathrm{V}_{\text {flux }}=0\right.$ for $\left.\mathrm{r} \rightarrow+\infty\right)$.

Definition of the Preferred Frame of Reference based on the entities constituting the medium:

Let us consider a Galilean referential R (a laboratory) and an elementary volume linked to this referential.

In this very small volume, imagine that we can count the entities in it (gravitons) and we can also know the speed vector of each graviton $\overrightarrow{V_{G / R}}$.

Knowing this, it is possible to compute the vectoral average of the speed vectors of the gravitons: $\overrightarrow{C_{G / R}}=\sum_{i=1}^{N_{G}} \overrightarrow{V_{G / R}} / N_{G}$.

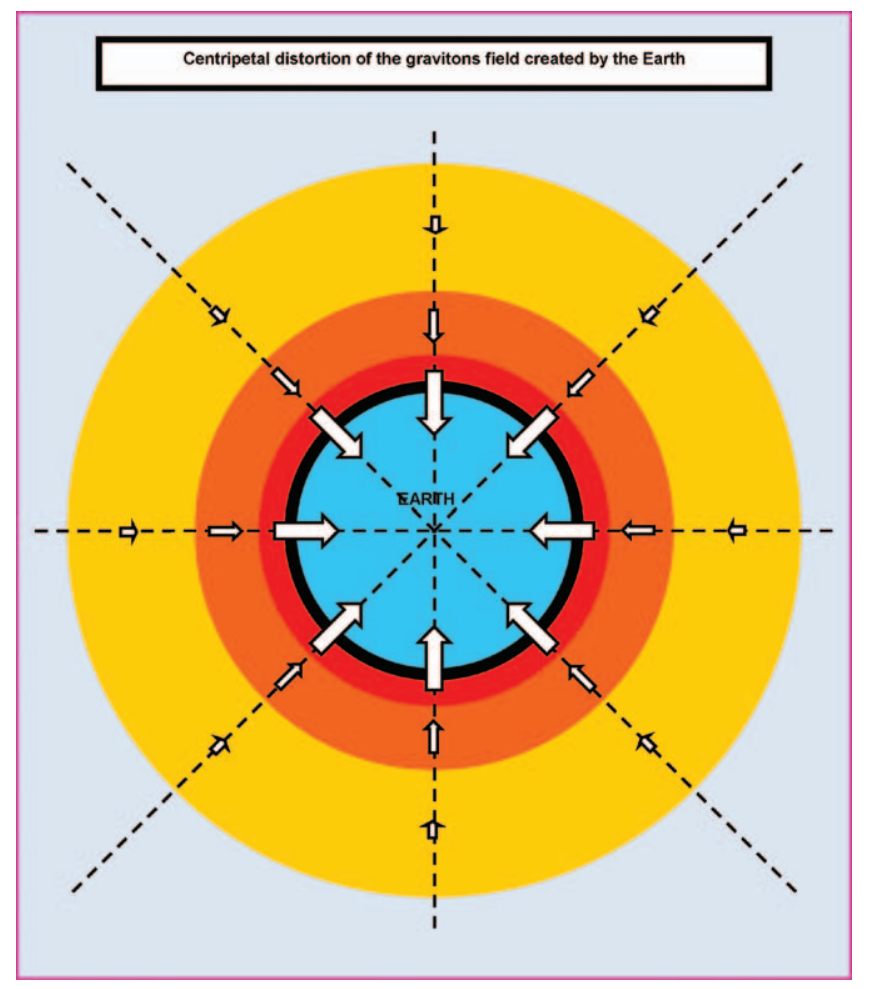

FIG. 3. Distortion of the gravitons field due to the Earth. 
This resultant vector means that, at the center of this given elementary volume, the Preferred Frame of Reference moves at the speed $\overrightarrow{C_{G / R}}$ versus the referential $\mathrm{R}$ and that the referential $\mathrm{R}$ (the laboratory) moves at the speed $-\overrightarrow{C_{G / R}}$ versus the Preferred Frame of Reference (defined by the medium), i.e., versus the medium.

Figure 3 shows the distortion of the gravitons field created by the Earth.

The distortion of the gravitons field (the flux of the medium):

- is generated by the presence of the matter of the Earth,

- is always centripetal, i.e., radial, oriented toward the center of gravity of the Earth,

- is maximum at the surface of the Earth and decreases when going away from the Earth,

- has a constant modulus on every sphere whose center is the one of the Earth,

- gives the impression to follow the Earth in its movement (whatever its speed) because it remains identical to itself.

\section{APPENDIX B: SPEED OF PROPAGATION OF GRAVITATION}

\section{Gravitational waves}

In the theory of general relativity, the gravitational waves are a small perturbation of space-time which propagates at the speed of light.

In the theory of the dynamic medium of reference, the gravitational waves are a small perturbation of the medium which propagates at the speed of light.

\section{Speed of propagation of gravitation}

In the theory of the dynamic medium of reference, the speed of gravitation (the physical effect which produces that an object falls toward a massive body) is the one of the gravitons introduced in the previous paragraph and is supposed to be much greater than the speed of light.

In the theory of the dynamic medium of reference, which is an extension of Lorentz-Poincaré's theory in the domain of gravitation, nothing prevents the existence of entities moving much faster than light.

It is only in Einstein's relativity that nothing can overtake the speed of light.

\section{Entanglement of two particles}

In The Ghost in the Atom, ${ }^{14}$ there is the following discussion about the EPR experiment and locality (page 39):

"If locality is abandoned, it is possible to re-create a description of the microworld closely similar to that of the everyday world, with objects having a concrete independent existence in well-defined states and possessing complete sets of physical attributes. No need for fuzziness now.

The trade-off is, of course, that nonlocal effects bring their own crop of difficulties; specifically, the ability for signals to travel backward into the past. This would open the way to all sorts of causal paradoxes."
It is essential to be aware that all these difficulties are only in Einstein's vision, i.e., the special relativity (and general relativity).

If one chooses the version of Lorentz and Poincaré, all these difficulties disappear.

In The Ghost in the Atom, ${ }^{14}$ John Bell says about the EPR paradox (page 48): "I would say, that the cheapest resolution is something like going back to relativity as it was before Einstein, when people like Lorentz and Poincaré thought that there was an aether-a preferred frame of reference-but that our measuring instruments were distorted by motion in such a way that we could not detect motion through the aether. Now, in that way one can imagine that there is a preferred frame of reference, and in this preferred frame of reference things do go faster than light."

In the same book, ${ }^{14}$ David Bohm says about locality: "I would be quite ready to relinquish locality; I think it is an arbitrary assumption." and Basil Hiley says about non locality: "If you have an absolute spacetime, or an absolute time, in the background then you don't get into causal loops. So, the causal paradoxes won't arise."

The entanglement of two particles (let us say, two photons) could be realized by the proposed gravitons which move much faster than light.

To determine the speed of gravitons, one reasoning is to consider that the connection between two particles remains even if the two particles are situated at two opposite extremities of our Universe and that our more accurate instruments cannot detect a duration between the measurement of the first particle and the measurement of the second particle, i.e., the duration is smaller than the Planck time $t_{\text {Planck }}=5.4 \times 10^{-44} \mathrm{~s}$.

So, we obtain the following speed for the gravitons and the speed of gravitation:

$$
V_{\text {gravitons }}=\frac{D_{\text {Universe }}}{t_{\text {Planck }}}=3 \times 10^{69} \mathrm{~m} / \mathrm{s} \text {. }
$$

It is only in Einstein's version (the theory of relativity) that nothing can move faster than light.

\section{Proposed experiment to measure the speed of propagation of gravitation}

The only way to be really sure of the speed of propagation of gravitation is a direct measure, that is to say, the effect of the modification of a first material system on a second material system including the modification of the distribution of matter, the modification of the distance between the two systems at a high speed.

The cosmos seems to be the best laboratory to measure the speed of propagation of gravitation.

For example, we can consider a gigantic triangle (system 1, system 2, Earth) and we suppose that system 1 undergoes a titanic and violent change (explosion, supernova, ...).

We suppose that the distances between the two systems and between the systems and the Earth are perfectly known.

We call « optical image » of an event, the light emitted or reflected by the object concerned by the event at the time of the event. This optical image propagates at the speed of light and can be received at different times by different observers. 
By knowing the time of arrival on Earth of the optical image of the violent phenomena which occurs in system 1 as well as the time of arrival on Earth of the optical image of the gravitational effects which affect system 2 , it is possible to deduce the speed of propagation of gravitation.

We call $d_{\mathrm{S} 1 \mathrm{E}}$ the distance between system 1 and the Earth, $\mathrm{d}_{\mathrm{S} 2 \mathrm{E}}$ the distance between system 2 and the Earth, $d_{\mathrm{S} 1 \mathrm{~S} 2}$ the distance between the two systems and $\mathrm{V}_{\mathrm{G}}$ the speed of propagation of gravitation.

We call $t_{1}$ the time when the violent event occurs in system $1, t_{1}^{\prime}$ the time of arrival on Earth of the optical image of this event, $t_{2}$ the beginning of the gravitational effects on system 2 and finally $t_{2}{ }^{\prime}$ the time of arrival on Earth of the optical image of this last event which occurs in system 2.

We have

$$
t_{1}^{\prime}=t_{1}+\frac{d_{S 1 E}}{c} \quad t_{2}=t_{1}+\frac{d_{S 1 S 2}}{V_{G}} \text { and } t_{2}^{\prime}=t_{2}+\frac{d_{S 2 E}}{c} .
$$

We can deduce

$$
\begin{aligned}
t_{2}^{\prime}-t_{1}^{\prime} & =t_{2}+\frac{d_{S 2 E}}{c}-\left(t_{1}+\frac{d_{S 1 E}}{c}\right) \\
& =t_{1}+\frac{d_{S 1 S 2}}{V_{G}}+\frac{d_{S 2 E}}{c}-\left(t_{1}+\frac{d_{S 1 E}}{c}\right) \\
& =\frac{d_{S 1 S 2}}{V_{G}}+\frac{d_{S 2 E}-d_{S 1 E}}{c} .
\end{aligned}
$$

Then we deduce the speed of propagation of gravitation

$$
V_{G}=d_{S 1 S 2} /\left(\Delta t^{\prime}-\frac{\Delta d}{c}\right) \text { with } \Delta \mathrm{t}^{\prime}=\mathrm{t}_{2}^{\prime}-\mathrm{t}_{1}^{\prime} \text { and } \Delta \mathrm{d}=\mathrm{d}_{\mathrm{S} 2 \mathrm{E}}-\mathrm{d}_{\mathrm{SIE}} \text {. }
$$

Particular cases

(1) $V_{G}=c$, then we have: $t_{2}^{\prime}-t_{1}^{\prime}=\left(d_{S 1 S 2} / c\right)+(\Delta d / c)$. It is the case of general relativity.

(2) $V_{G} \ggg c$, then we have: $t_{2}^{\prime}-t_{1}^{\prime} \approx \Delta d / c$. It is the case of the theory of the dynamic medium of reference.
Obviously, the bigger the distance $d_{\mathrm{S} 1 \mathrm{~S} 2}$ will be, the more definitive it will be to decide between general relativity and the theory of the dynamic medium of reference.

Other proposition of experiment with swift massive bodies:

We can also envisage the measure of the gravitational effect of a massive body, moving at a speed as close as possible to the speed of light, on a second massive body.

The first massive body would start at a long distance from the second body, where its gravitational effect is negligible, and then would pass very close to the second body at a very high speed.

If the measure is possible, we could see if the gravitational effect propagates at the speed of light or much faster.

${ }^{1} \mathrm{C}$. Will, Theory and Experiment in Gravitational Physics (Cambridge University Press, Cambridge, 1993).

${ }^{2} \mathrm{C}$. Will, Was Einstein Right? Putting General Relativity to the Test (Basic Books, New York, 1993).

${ }^{3}$ J.-C. Boudenot, Électromagnétisme et Gravitation Relativistes (Ellipses, Étuz, France, 1989).

${ }^{4} \mathrm{H}$. Poincaré, Science et Méthode (Flammarion, Paris, France, 1908).

${ }^{5} \mathrm{M}$. Lambert, Relativité Restreinte Et Electromagnétisme (Ellipses, Étuz, France, 2000).

${ }^{6} \mathrm{~J} . \mathrm{S}$. Bell, Speakable and Unspeakable in Quantum Mechanics (Cambridge University Press, Cambridge, 1987).

${ }^{7}$ J. Gribbin, Schrödinger's Kittens and the Search for Reality (Phoenix International Publications, Phoenix, AZ, 1996).

${ }^{8}$ L. Smolin, Time Reborn (Houghton Mifflin Harcourt, San Diego, CA, 2013).

${ }^{9} \mathrm{~A}$. Barrau and J. Grain, Relativité Générale (Dunod, Paris, France, 2011).

${ }^{10}$ A. Einstein, Ann. Phys. 35, 898 (1911).

${ }^{11}$ M. Holson, G. Efstathiou, and A. Lasenby, Relativité Générale (De Boeck, Paris, France, 2010).

${ }^{12}$ A. Einstein, La Théorie de la Relativité Restreinte et Générale (Dunod, Paris, France, 2004).

${ }^{13}$ B. Greene, The Fabric of the Cosmos (Penguin Books, 2008).

${ }^{14} \mathrm{P}$. Davies and J. Brown, The Ghost in the Atom (Cambridge University Press, Cambridge, 2000). 